\title{
Analysis of Communication Errors During on-pump Coronary Artery Bypass Grafting (CABG)
}

Keiko Okuyama $^{1}$, Sharil Azlan Ariffin ${ }^{1}$

Shunichi Takagi ${ }^{2}$, Hideyuki Higuchi ${ }^{2}$

Department of Anaesthesiology, National Heart Institute of Malaysia

Department of Anaesthesiology, Tokyo Women's Medical University, Japan²

\section{INTRODUCTION \& AIM}

- Communication failures are commonly accepted as the most important causative factor of adverse events during surgery.

- Cardiac surgery, especially, requires numerous professionals to communicate to each other. However, communication status in common cardiac surgery is unknown.

- The aim of this study was to investigate communication errors in on-pump coronary artery bypass grafting (CABG) operations, which is one of the most common open heart procedures in adult cardiac surgery, such that surgical staff are generally familiar with the procedure.

\section{METHODS}

- 50 elective conventional on-pump coronary artery bypass grafting (CABG) procedures were observed at National Heart Institute, Malaysia.

- Over 3 months period (May 2014 - July 2014)

- Communication errors were recorded by an anaesthetic fellow from the administration of heparin to chest closure.

- Types, subjects and details of errors were recorded such that they could be analyse using communication failure types ${ }^{1}$ and human error classification ${ }^{2}$ (Table 1, 2)

\begin{tabular}{|c|c|}
\hline \multicolumn{2}{|c|}{ Types of Communication Failure1 (Table 1) } \\
\hline Occasion failures & $\begin{array}{c}\text { Problems in the situation or context of the } \\
\text { communication event }\end{array}$ \\
\hline Content failures & $\begin{array}{c}\text { Insufficiency or inaccuracy apparent in the information } \\
\text { being transferred }\end{array}$ \\
\hline Audience failures & $\begin{array}{c}\text { Gaps in the composition of the group engaged in the } \\
\text { communication }\end{array}$ \\
\hline Purpose failures & $\begin{array}{c}\text { Communication events in which purpose is unclear, not } \\
\text { achieved, or inappropriate }\end{array}$ \\
\hline
\end{tabular}

\begin{tabular}{|c|c|}
\hline \multicolumn{2}{|c|}{ Human Error Classification ${ }^{2}$ (Table 2) } \\
\hline Mistakes & Errors brought by a faulty plan/intention \\
\hline Slips & Actions not carried out as intended or planned \\
\hline Lapses & Missed actions and omissions \\
\hline Violations & Deliberate omissions \\
\hline
\end{tabular}

\section{RESULTS}

(Table 3)

- A total of 119 errors were recorded.

- Mean: 2.38 errors per case

- Mode: $1(\mathrm{~N}=16)$

- Minimum 0, maximum 9 errors (No error was found in 6 cases $(12 \%)$ )
90

70

60

50

40

30

20

10
Communication Failure Types (No. of Error)

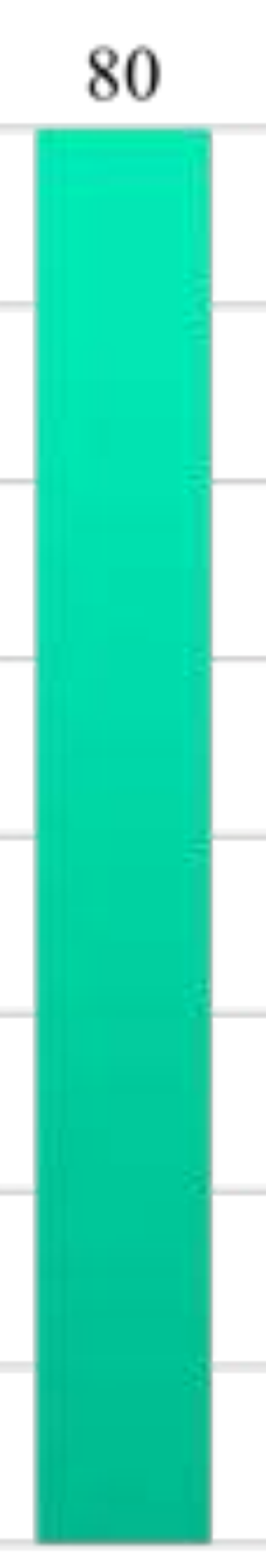

purpose
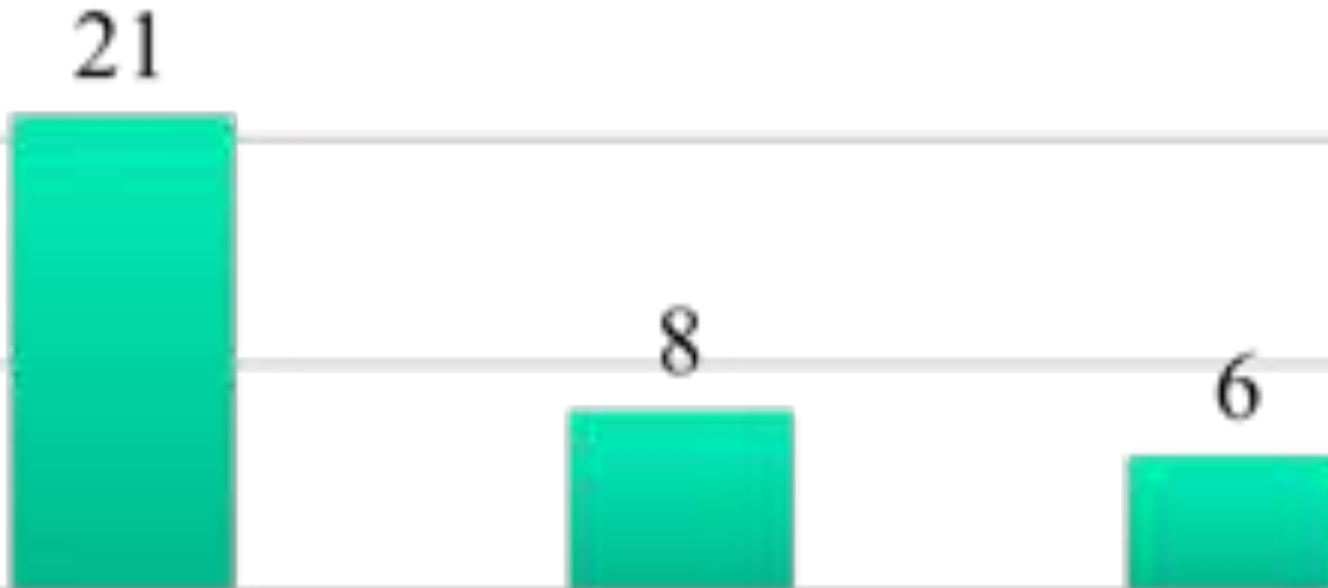

occasion audience (Figure 1)

\begin{tabular}{|lcc|}
$\begin{array}{l}\text { Characteristics of Error- } \\
\text { Involved Participants }\end{array}$ & $\begin{array}{l}\text { No. of } \\
\text { errors }\end{array}$ & $\%$ \\
\hline Seniors & 102 & 85.7 \\
\hline Juniors & 51 & 42.9 \\
\hline Physicians & 112 & 94.1 \\
\hline Non-Physicians & 77 & 64.7 \\
\hline
\end{tabular}

Human Error Classification(No. of Error)

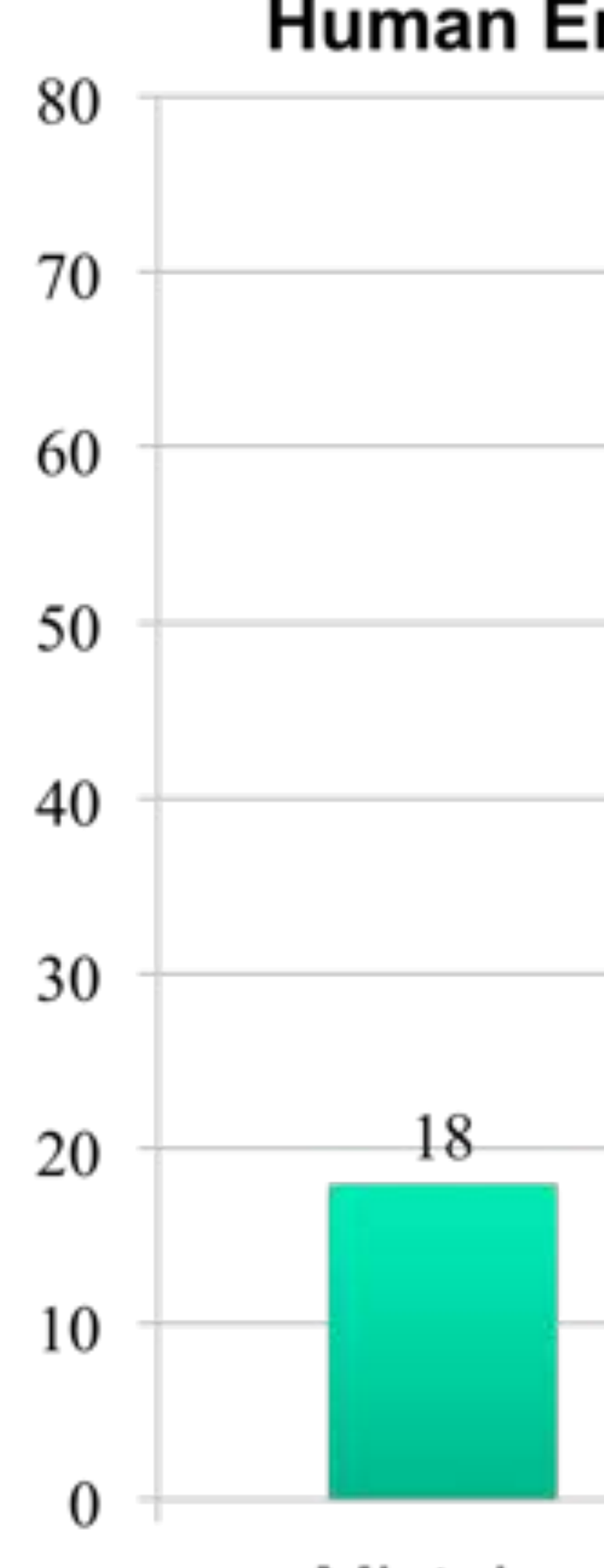

Mistake

\section{DISCUSSION}

- Surgeons were more involved in communication errors in cardiac surgery (Table 4). It is assumed that most communication involved surgeons during surgical period.

- Slip/violation errors were found in approximately half of CABG cases, which involved both senior and junior members.

- Violation errors may happen when; (i) juniors hesitate to speak up to seniors/other professionals, (ii) seniors assume they do not have to do it.

- Most errors happened in common/uncomplicated situations.

- Assessing the surgical team culture followed by implementation of teamwork improvement programmes may be useful to encourage surgical staff to speak up and to prevent latent adverse event in the operating theatre.
(Table 4)

\begin{tabular}{|lcc|} 
Error-Involved Professionals & $\begin{array}{c}\text { No. of } \\
\text { errors }\end{array}$ & $\%$ \\
\hline Anaesthetists & 45 & 37.8 \\
\hline Surgeons & 90 & 75.6 \\
\hline Perfusionists & 47 & 39.5 \\
\hline Scrub nurses & 17 & 14.3 \\
\hline Circulating nurses & 14 & 11.8 \\
\hline
\end{tabular}

- The most common mistake error was scrub nurses' handing a wrong instrument to surgeons.

- The most common lapse error was repeating or being misunderstood of order/questions by surgeons.

- Junior members caused more violation/slip errors than seniors

(56.5\% vs. $43.5 \%)$

- Physicians were more involved in violation/slip errors (74.1\%)

- 2 slip/violation errors by nonphysicians could have caused serious accidents.

- More than $80 \%$ of total error happened with standerdised phrases $(93,78.2 \%$ vs. $22,18.5 \%)$

\section{Lapse (Figure 2) \\ Slip/Violation}

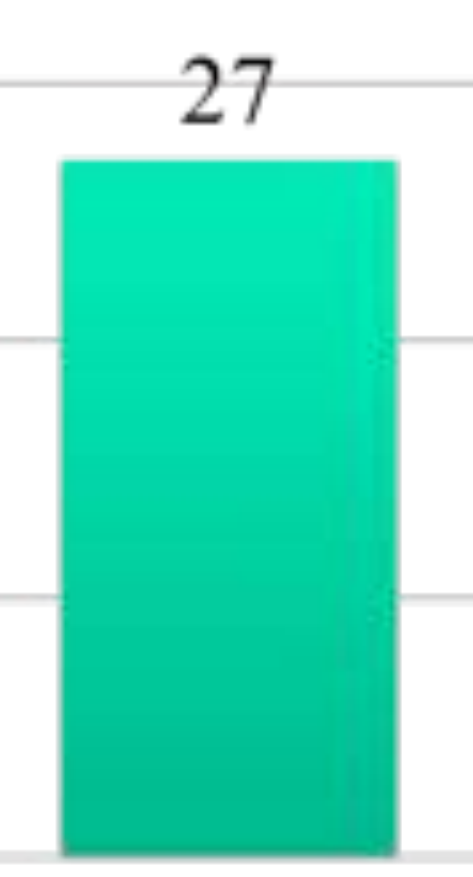

\section{CONCLUSION}

- There were a number of communication errors found with routine work in one of the most common cardiac surgery operations.

- Slip/violation errors happened among surgical staff without attention.

\section{REFERENCES}

1. Lingard, Lorelei, et al. "Communication failures in the operating room: an observational classification of recurrent types and effects." Quality and Safety in Health Care 13.5 (2004): 330-334.

2. Reason, James. Human error. Cambridge university press, 1990. 